\title{
Influence of functional groups on charge transport in molecular junctions
}

\author{
Mowbray, Duncan; Jones, Glenn; Thygesen, Kristian Sommer
}

Published in:

Journal of Chemical Physics

Link to article, DOI:

$10.1063 / 1.2894544$

Publication date:

2008

Document Version

Publisher's PDF, also known as Version of record

Link back to DTU Orbit

Citation (APA):

Mowbray, D., jones, G., \& Thygesen, K. S. (2008). Influence of functional groups on charge transport in molecular junctions. Journal of Chemical Physics, 128(11), 111103. https://doi.org/10.1063/1.2894544

\section{General rights}

Copyright and moral rights for the publications made accessible in the public portal are retained by the authors and/or other copyright owners and it is a condition of accessing publications that users recognise and abide by the legal requirements associated with these rights.

- Users may download and print one copy of any publication from the public portal for the purpose of private study or research.

- You may not further distribute the material or use it for any profit-making activity or commercial gain

- You may freely distribute the URL identifying the publication in the public portal 


\title{
Influence of functional groups on charge transport in molecular junctions
}

\author{
D. J. Mowbray, ${ }^{\text {a) }}$ G. Jones, and K. S. Thygesen ${ }^{\text {b) }}$ \\ Center for Atomic-scale Materials Design (CAMD), Department of Physics, Technical University \\ of Denmark, DK-2800 Kgs. Lyngby, Denmark
}

(Received 3 December 2007; accepted 14 February 2008; published online 18 March 2008)

\begin{abstract}
Using density functional theory (DFT), we analyze the influence of five classes of functional groups, as exemplified by $\mathrm{NO}_{2}, \mathrm{OCH}_{3}, \mathrm{CH}_{3}, \mathrm{CCl}_{3}$, and $\mathrm{I}$, on the transport properties of a 1,4-benzenedithiolate (BDT) and 1,4-benzenediamine (BDA) molecular junction with gold electrodes. Our analysis demonstrates how ideas from functional group chemistry may be used to engineer a molecule's transport properties, as was shown experimentally and using a semiempirical model for BDA [Nano Lett. 7, 502 (2007)]. In particular, we show that the qualitative change in conductance due to a given functional group can be predicted from its known electronic effect (whether it is $\sigma / \pi$ donating/withdrawing). However, the influence of functional groups on a molecule's conductance is very weak, as was also found in the BDA experiments. The calculated DFT conductances for the BDA species are five times larger than the experimental values, but good agreement is obtained after correcting for self-interaction and image charge effects. (C) 2008 American Institute of Physics. [DOI: 10.1063/1.2894544]
\end{abstract}

One of the main advantages of molecular based electronics over present day semiconductor technology is the vast flexibility in design and functionality offered by the myriad of available molecules. ${ }^{1}$ In order to fully exploit this flexibility for the synthesis of molecular devices, it is vital to establish simple guiding rules to estimate the effect that a given change in the molecule's composition or conformation has on its electrical properties.

So far, most experimental and theoretical work on single-molecule conduction has focused on understanding the basic properties of individual junctions. ${ }^{2-5}$ On the other hand, few studies have aimed at describing general trends across molecular species. ${ }^{6-8}$

It is well known that the chemistry of a functional parent or parent molecule may be altered in a consistent manner by the attachment of functional groups. ${ }^{9}$ Further, the influence of a particular functional group on a parent molecule's chemistry may be predicted qualitatively by considering the electronic effect of the functional group.

We will demonstrate how such ideas may also be applied to describe the influence of a functional group on a parent molecule's transport properties. This was recently shown experimentally for 1,4-benzenediamine (BDA), and the results were explained by scaling the molecule's empirical conductance value by the calculated ionization potentials in the gas phase for the BDA species with two bonded Au atoms. ${ }^{10} \mathrm{By}$ employing these concepts from functional group chemistry, we may engineer the electronic transport properties of a known functional parent. This may be done using a functional group whose frontier orbitals have the same symmetry as the conducting orbital of the parent molecule $(\sigma$ or $\pi)$. Groups with donating/withdrawing electronic effects may

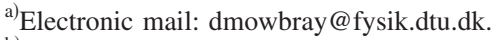

${ }^{b)}$ Electronic mail: thygesen@fysik.dtu.dk.
}

then be used to raise/lower the eigenenergy of the conducting orbital.

In this paper, we analyze the influence of functional groups on the conductance of both a 1,4-benzenedithiolate $\left(\mathrm{C}_{6} \mathrm{H}_{4} \mathrm{~S}_{2}\right.$ or BDT) and a 1,4-benzenediamine $\left(\mathrm{C}_{6} \mathrm{H}_{4}\left(\mathrm{NH}_{2}\right)_{2}\right.$ or BDA) molecular junction between gold contacts. We consider these molecules because BDT is perhaps the best studied molecule for charge transport, ${ }^{2,3,11}$ while the influence of functional groups on the BDA molecule has been recently studied experimentally. ${ }^{10}$ In particular, we shall consider prototypical functional groups from each of the five electronic categories, as shown in Table I. These categories are (1) withdrawal by conjugation through the $\pi$ network ( $\pi$ withdrawal), (2) donation by conjugation through the $\pi$ network ( $\pi$ donation), (3) donation by inductive effect through the $\sigma$ network ( $\sigma$ donation), (4) withdrawal by inductive effect through the $\sigma$ network ( $\sigma$ withdrawal), and (5) withdrawal by inductive effect through the $\sigma$ network with donation by conjugation through the $\pi$ network ( $\sigma$ withdrawal and $\pi$ donation). ${ }^{9}$ For each electronic effect categorization, we have selected as respective examples a nitro group $\left(\mathrm{NO}_{2}\right)$, a methoxy group $\left(\mathrm{OCH}_{3}\right)$, a methyl group $\left(\mathrm{CH}_{3}\right)$, a trichloromethyl group $\left(\mathrm{CCl}_{3}\right)$, and an iodo group (I). Other examples of functional groups from each category are also provided in Table I.

All calculations have been performed using the SIESTA density functional theory (DFT) code $^{12}$ with the PBE exchange correlation $(\mathrm{xc})$ functional, ${ }^{13}$ a double zeta polarized (DZP) basis set, and a mesh cutoff of 200 Ry. We first adsorbed the molecules on a five layer thick gold (111) slab and relaxed the molecule and the three outermost surface layers. The supercell contained $3 \times 3$ gold atoms in the surface plane, within which we used $2 \times 2 k$ points. A pyramid of four gold atoms attached to the five layers of gold (111) was then introduced in the supercell to simulate the tip of a scanning tunneling microscope (STM). The pyramid was placed 
TABLE I. Functional group categorization by electronic effect with further examples (Ref. 9).

\begin{tabular}{|c|c|c|c|c|}
\hline \multicolumn{2}{|r|}{$\begin{array}{c}\text { Functional } \\
\text { Group }\end{array}$} & Structure & $\begin{array}{c}\text { Electronic } \\
\text { Effect }\end{array}$ & $\begin{array}{c}\text { Other } \\
\text { Examples }\end{array}$ \\
\hline $\mathrm{NO}_{2}$ & nitro & & $\pi$ withdrawal & $\begin{array}{c}\mathrm{CN}, \mathrm{COR}, \\
\mathrm{SO}_{3} \mathrm{R}\end{array}$ \\
\hline $\mathrm{OCH}_{3}$ & methoxy & & $\pi$ donation & $\mathrm{NR}_{2}, \mathrm{OR}$ \\
\hline $\mathrm{CH}_{3}$ & methyl & & $\sigma$ donation & $\begin{array}{c}\mathrm{C}_{2} \mathrm{H}_{5}, \mathrm{C}_{3} \mathrm{H}_{7} \\
\text { alkyls }\end{array}$ \\
\hline $\mathrm{CCl}_{3}$ & trichloromethyl & & $\sigma$ withdrawal & $\mathrm{CF}_{3}, \mathrm{NR}_{3}^{+}$ \\
\hline I & iodo & & $\begin{array}{l}\sigma \text { withdrawal \& } \\
\pi \text { donation }\end{array}$ & $\begin{array}{l}\mathrm{F}, \mathrm{Cl}, \mathrm{Br} \\
\text { halogens }\end{array}$ \\
\hline
\end{tabular}

with its apex atom close to the unbound $\mathrm{S} / \mathrm{N}$ atom of the molecule, and upon further relaxation, the molecule bonds to the gold apex atom, as shown in Fig. 1.

In this way, we model a low temperature conductance measurement for a low molecular coverage on a gold (111) surface using a STM tip. ${ }^{14}$ Such a configuration may not accurately describe the results of break-junction experiments, especially for the weakly bound BDA species. ${ }^{15}$ However, as shown in Ref. 15, such a binding site yields a similar transmission function to more energetically favorable geometries. Thus, our chosen geometry should allow us to discern more broadly applicable trends in the electronic effects of the functional groups, which are our primary interest.

We find that the BDT species (BDT, $\mathrm{NO}_{2} \mathrm{BDT}$, $\mathrm{OCH}_{3} \mathrm{BDT}, \mathrm{CH}_{3} \mathrm{BDT}$, and I BDT) prefer the bridge site of the (111) surface and are rotated by approximately $30^{\circ}$ to the surface normal, ${ }^{16}$ as shown in Fig. 1(a). On the other hand, we find that the $\mathrm{BDA}$ species $\left(\mathrm{BDA}, \mathrm{NO}_{2} \mathrm{BDA}, \mathrm{OCH}_{3} \mathrm{BDA}\right.$,
$\mathrm{CH}_{3} \mathrm{BDA}$, and I BDA) prefer the atop site and lie down at an angle of approximately $50^{\circ}$ to the surface normal, ${ }^{15}$ as shown in Fig. 1(b).

Having chosen the contact geometries, we calculated the elastic transmission functions using the nonequilibrium Green's function formalism. The calculation procedure is equivalent to the one described in Ref. 17, except that the Hamiltonian was represented in the SIESTA atomic orbital basis instead of Wannier functions. We note here that the DZP SIESTA basis set has recently been shown to yield transmission functions in quantitative agreement with plane wave codes and maximally localized Wannier functions. ${ }^{18}$ The transmission function was averaged over four $k$ points in the surface plane, and three gold (111) layers were added on both sides of the molecule before the system was coupled to the bulk leads to ensure a smooth matching of the effective DFT potential.

The calculated transmission functions are shown in Fig. 1. For both BDT and BDA, the change in the transmission function, $T(E)$, near the Fermi level when the functional groups are attached, is strikingly small.

The calculated conductances, $G=G_{0} T\left(E_{F}\right)$, where $G_{0}$ $=2 e^{2} / h$, for the BDT and BDA species are shown in Table II. As is often the case for DFT transport calculations on molecular contacts, our results differ substantially from the experimental values of $0.011 G_{0}$ obtained for BDT (Ref. 11) and $0.0064 G_{0}$ for BDA. ${ }^{15} \mathrm{We}$ will address this issue in the last part of the paper. At this point, we observe that functional groups whose valence orbitals are of $\pi$ symmetry seem to have the most influence on the molecule's conductance, with the $\pi$ withdrawing group lowering the conductance and the $\pi$ donating group raising the conductance.

For both BDT and BDA species, we found that transport primarily occurs through the highest occupied molecular orbital (HOMO), which in both cases is a $\pi$ bonding molecular (a)

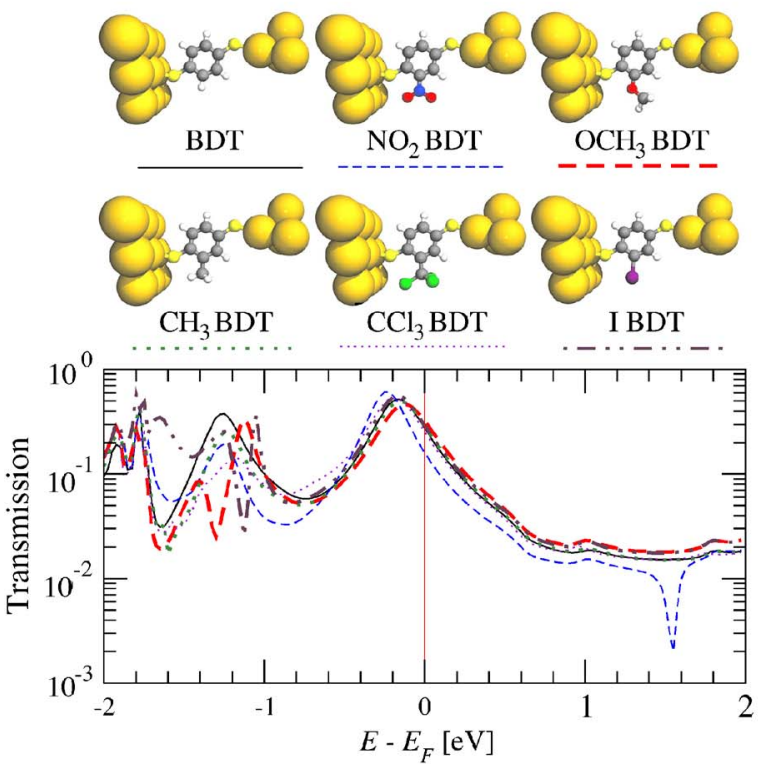

(b)
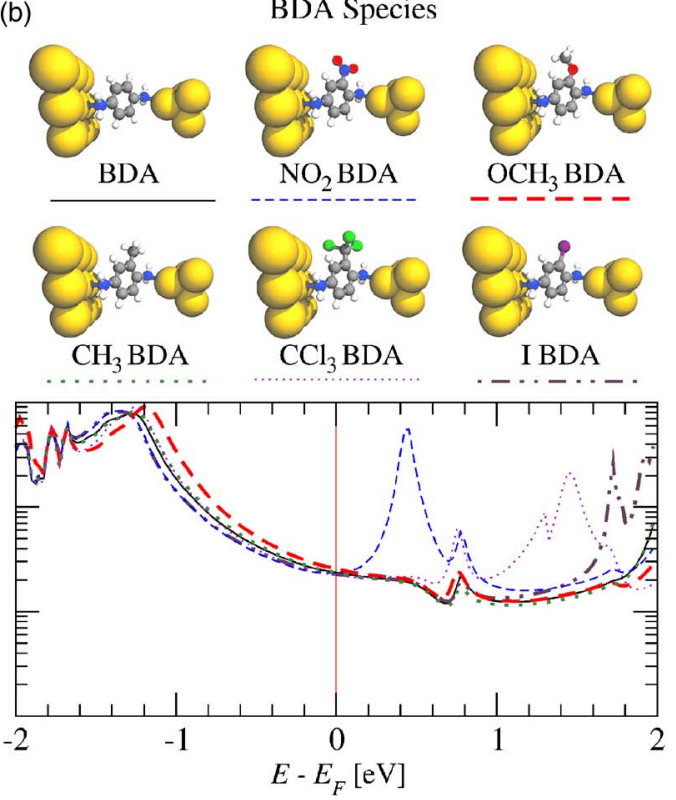

FIG. 1. (Color online) Schematics of (a) BDT species and (b) BDA species bonded between a gold (111) surface and tip, and their transmission functions vs energy $E-E_{F}$ in eV relative to the metal's Fermi energy $E_{F}$. 
TABLE II. Conductance $G$ of BDT and BDA species between a gold (111) surface and tip.

\begin{tabular}{lclc}
\hline \hline BDT species & $G\left(2 e^{2} / h\right)$ & BDA species & $G\left(2 e^{2} / h\right)$ \\
\hline BDT & 0.28 & BDA & 0.024 \\
$\mathrm{NO}_{2}$ BDT & 0.16 & $\mathrm{NO}_{2}$ BDA & 0.024 \\
$\mathrm{OCH}_{3}$ BDT & 0.32 & $\mathrm{OCH}_{3}$ BDA & 0.026 \\
$\mathrm{CH}_{3} \mathrm{BDT}$ & 0.29 & $\mathrm{CH}_{3} \mathrm{BDA}$ & 0.024 \\
$\mathrm{CCl}_{3}$ BDT & 0.25 & $\mathrm{CCl}_{3} \mathrm{BDA}$ & 0.022 \\
$\mathrm{I} \mathrm{BDT}$ & 0.29 & $\mathrm{I} \mathrm{BDA}$ & 0.023 \\
\hline \hline
\end{tabular}

orbital, as shown in Fig. 2. In principle, the concept of molecular orbitals is somewhat artificial for a chemisorbed molecule, as hybridization effects will broaden the levels into resonances. One way of generalizing the concept is to consider $\hat{H}_{\text {mol }}$, the projection of the Hamiltonian of the contacted system onto the subspace spanned by the basis functions of the molecule. The eigenvectors of $\hat{H}_{\text {mol }}$ can be regarded as molecular orbitals renormalized by the electrodes. ${ }^{17}$ For molecules which are not too strongly coupled to the electrodes, these renormalized molecular levels are easily identified with the levels of the free molecule.

In Fig. 2, we compare the position of the HOMO level (relative to $E_{F}$ ) of the contacted molecules with the HOMO level (relative to vacuum) of the free gas phase molecules. The variation in the HOMO position with the functional group correlates directly with the variation in the conductance (see Table II), which shows that the current is indeed carried by the HOMO. One exception from this trend is $\mathrm{NO}_{2} \mathrm{BDA}$, for which the conductance is not lowered even though the HOMO is significantly downshifted. This is because the lowest-unoccupied molecular orbital (LUMO) of $\mathrm{NO}_{2} \mathrm{BDA}$ lies so low that it also contributes to the charge transport. From Fig. 2, we can also see that each functional group produces a remarkably consistent shift of the conducting orbital for BDA and BDT. This agrees with the basic premise of functional group chemistry, that a given functional group will alter different parent molecules in a consistent way. We also notice that the two $\pi$ groups $\left(\mathrm{NO}_{2}\right.$ and $\mathrm{OCH}_{3}$ ) give the largest shifts of the eigenenergies, while the functional groups with $\sigma$ symmetry have little influence. Moreover, the $\pi$ withdrawing functional group $\left(\mathrm{NO}_{2}\right)$ delocalizes the HOMO and thereby lowers the eigenenergy, while the $\pi$ donating functional group $\left(\mathrm{OCH}_{3}\right)$ confines the HOMO and thereby increases the eigenenergy. Thus, the main ideas of functional group chemistry may be employed to qualitatively predict a functional group's influence on a given functional parent's conducting orbital(s).

We stress that the qualitative effect of the functional groups on the HOMO of the contacted molecule, and thus its conductance, roughly follows from the effect of the functional groups on the free molecule's HOMO, as was found experimentally for BDA. ${ }^{10}$ This is significant, as it suggests that the latter could be used as a simple descriptor to estimate the impact of a given functional group on the conductance, thereby allowing for an efficient screening of large numbers of functional groups.

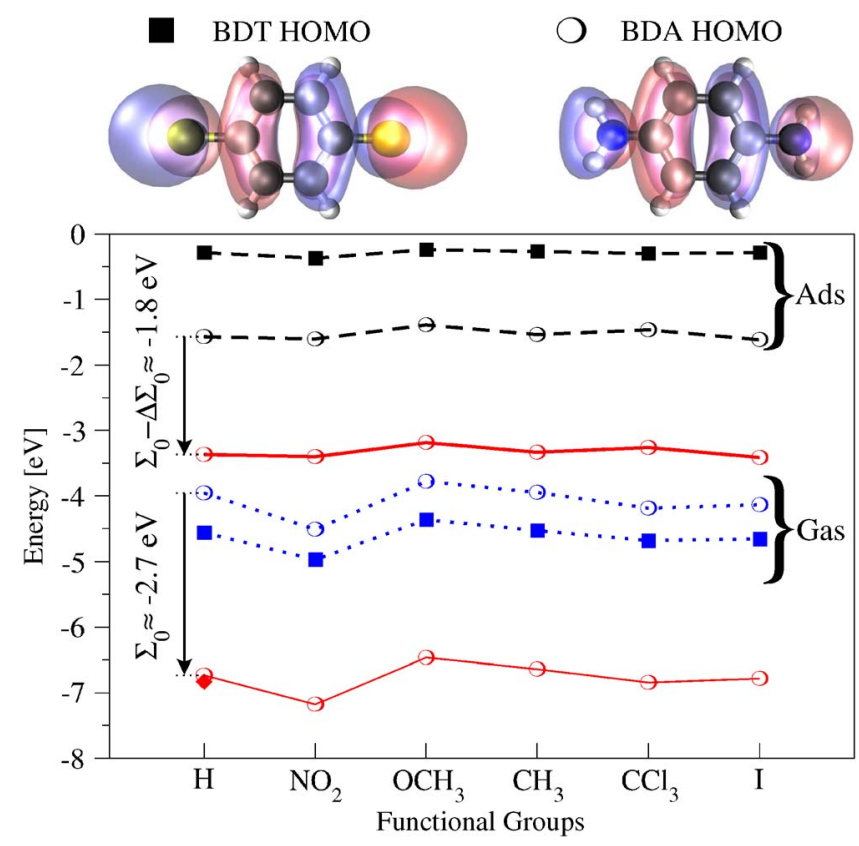

FIG. 2. (Color online) Eigenenergies in $\mathrm{eV}$ and isosurfaces of $\pm 0.02 e / \AA^{3}$ of HOMOs for BDT species (squares) and BDA species (circles) when adsorbed between a gold (111) surface and tip relative to the metal's Fermi level $E_{F}$ (black dashed lines) and in the gas phase relative to vacuum (blue dotted lines). For BDA species, the experimental (diamond) and calculated (thin red solid line) gas phase ionization potential $I_{0}$ with a SO shift of $\Sigma_{0} \approx-2.7 \mathrm{eV}$ and the eigenenergies in the junction with a SO shift of $\Sigma_{0}+\Delta \Sigma_{0} \approx-1.8 \mathrm{eV}$ (thick red solid line) are shown.

When we compare in Fig. 2 the magnitude of the shifts due to the functional groups for both BDT and BDA, we find significantly smaller effects for the contacted molecules than for the gas phase molecules. For example, the shift due to $\mathrm{NO}_{2}$ on BDT is approximately $0.4 \mathrm{eV}$ in the gas phase, while it is less than $0.1 \mathrm{eV}$ in the contact. This may be understood by recognizing that the gold contacts act as electron sources/ sinks for the parent molecule, counteracting the functional group's electronic influence. We thus believe that the weak influence of the functional groups on the conductance is a result of the self-consistent "pinning" of the HOMO level, which ensures the charge neutrality of the molecule. Such effects are indeed physical but could be artificially enhanced by self-interaction errors in the PBE xc functional: For partially occupied molecular orbitals, an incomplete cancellation of the Coulomb self-interaction by the xc functional will artificially raise (lower) the energy of that orbital when charge is added (removed) and this will enhance the "level pinning."

Self-interaction errors also contribute to the well-known underestimation of band gaps by DFT calculations. ${ }^{19}$ Recently, an atomic self-interaction correction (SIC) scheme $\mathrm{e}^{20,21}$ has been proposed as a simple cure to this problem for molecular contacts. In general, however, image charges formed in the metallic electrodes when electrons are added to or removed from the molecule also renormalize the molecular levels, ${ }^{22}$ and this effect is not captured by the SIC.

In Table III, we compare our DFT calculated conductances for the BDA species with corresponding experimental values. ${ }^{10}$ The calculated numbers are roughly five times larger than the experimental ones due to the above men- 
TABLE III. Calculated conductance $G_{\text {calc }}$, SO conductance $G_{\text {SO }}$ (Ref. 15) with shift $\Sigma_{0}-\Delta \Sigma_{0} \approx-1.8 \mathrm{eV}$, and experimental conductance $G_{\text {expt }}$ (Ref. 10) for BDA species between a gold (111) surface and tip.

\begin{tabular}{lccc}
\hline \hline BDA species & $G_{\text {calc }}\left(2 e^{2} / h\right)$ & $G_{\text {SO }}\left(2 e^{2} / h\right)$ & $G_{\text {expt }}\left(2 e^{2} / h\right)$ \\
\hline BDA & 0.024 & 0.0035 & $0.0064^{\mathrm{a}}$ \\
$\mathrm{NO}_{2} \mathrm{BDA}$ & 0.024 & 0.0032 & $\cdots$ \\
$\mathrm{OCH}_{2} \mathrm{BDA}$ & 0.026 & 0.0035 & $0.0069^{\mathrm{a}}$ \\
$\mathrm{CH}_{3} \mathrm{BDA}$ & 0.024 & 0.0034 & $0.0064^{\mathrm{a}}$ \\
$\mathrm{CCl}_{3} \mathrm{BDA}$ & 0.022 & 0.0034 & $\cdots$ \\
$\mathrm{Cl}$ BDA & 0.015 & 0.0025 & $0.0060^{\mathrm{a}}$ \\
$\mathrm{I}$ BDA & 0.023 & 0.0036 & $\cdots$ \\
\hline \hline
\end{tabular}

${ }^{\mathrm{a}}$ Reference 10 .

tioned deficiencies of the DFT approach. It is, however, interesting to notice that (i) the weak effect of functional groups is found in both data sets and (ii) the qualitative effect of the functional groups on the conductance (increase/ decrease) is reproduced by calculations. In the second column of Table III, we present the results of our conductance calculations after a "scissors operator" (SO), which corrects for self-interaction errors and image charge effects, has been applied to the molecule's spectrum.

To construct the SO, we follow a recent work by Quek et al. who applied the scheme to a BDA-gold junction. ${ }^{15}$ In this method, the underestimation of the DFT HOMO and LUMO is corrected by shifting the occupied/unoccupied orbitals of the contacted molecule by $\Sigma_{0}^{o, v}= \pm \Sigma_{0}=\mp\left(I_{0}+\varepsilon_{\mathrm{HOMO}}\right)$, where $\varepsilon_{\text {HOMO }}$ is the DFT HOMO level for the free molecule and $I_{0}$ the ionization potential. ${ }^{23}$ We also calculate the latter in DFT from $I_{0}=E_{q=+1}-E_{q=0}$, where $E_{q=+1}$ is the total energy of the molecule with charge $+e$ in the gas phase, while $E_{q=0}$ is the total energy in the gas phase of the neutral molecule. For BDA without functional groups, we find $I_{0} \approx 6.73 \mathrm{eV}$, which agrees quantitatively with the experimental value of $6.83 \mathrm{eV} .{ }^{15}$ We found only small variations in $\Sigma_{0}$ for the different BDA species and have used $\Sigma_{0} \approx-2.7 \mathrm{eV}$ for all molecules, as indicated in Fig. 2.

To estimate the shift of the molecular levels by image charge effects, we first calculate the charge distribution after removing an electron from the free molecule. A Mulliken analysis is then used to approximate this continuous charge distribution by point charges located at the atoms of the molecule. We model the gold contacts as two perfectly conducting surfaces separated by $13.2 \AA$, which corresponds to the vacuum separation between the opposing gold (111) surfaces. We then obtain an image potential $\Delta \Sigma_{0} \approx-0.9 \mathrm{eV}$ for all of the BDA species considered. ${ }^{24}$ For the BDA molecular junctions, we thus shift all occupied/unoccupied orbitals (obtained by diagonalizing $\left.\hat{H}_{\text {mol }}\right)$ by $\Sigma_{0}^{o, u}-\Delta \Sigma_{0}^{o, u}= \pm\left(\Sigma_{0}-\Delta \Sigma_{0}\right)$ $\approx \mp 1.8 \mathrm{eV}$, as indicated in Fig. 2. Calculating the conductance using the renormalized Hamiltonian yields the values shown in Table III. We find that this ad hoc correction produces results approximately half those obtained from breakjunction experiments on the BDA species. ${ }^{10}$ This is most probably due to our choice of contact geometry, as our calculated conductance for BDA of $0.024 G_{0}$ is about half the average DFT value calculated over 15 different breakjunction contact geometries of $0.046 G_{0} \cdot{ }^{15}$ We stress, how- ever, that the use of this SO can only be justified for weakly coupled molecules with HOMO/LUMO levels well separated from $E_{F}$. In Fig. 2, we see that this is the case for the BDA species, since the HOMOs are localized near the amine contact groups, providing a poor overlap with the gold contact orbitals. However, for the BDT species, whose HOMOs are rather diffuse around the sulfur atoms, there is a strong overlap with the gold contact orbitals, so that application of the SO is not justified.

In conclusion, we found that the ideas of functional group chemistry may be applied to qualitatively predict the influence of a functional group on the electronic structure of a parent molecule, as has been found for BDA both experimentally and using a semiempirical model. ${ }^{10}$ However, we also found that functional groups have a very weak influence on a molecule's conductance, in agreement with recent experiments. ${ }^{10}$ The reason for the weak influence is that charge neutrality pins the HOMO/LUMO molecular levels, making it difficult to shift them relative to $E_{F}$. By applying multiple functional groups to the same parent molecule, it may be possible to obtain a stronger influence, as was found for BDA. ${ }^{10}$ By employing a scissors operator correcting for self-interaction errors and image charge effects, we obtained qualitative agreement with experimental conductance values for the BDA species. Our results suggest that effective "switching" of a molecule's conductance may require a more direct change in the strength or geometry of the molecule's contacts to overcome the level pinning of the metal.

We thank J. K. Nørskov, S. Dobrin, and M. Strange for useful discussions. The authors acknowledge support from the Danish Center for Scientific Computing through Grant No. HDW-1103-06. The Center for Atomic-scale Materials Design (CAMD) is sponsored by the Lundbeck Foundation.

${ }^{1}$ C. Joachim, J. K. Gimzewski, and A. Aviram, Nature (London) 408, 541 (2000).

${ }^{2}$ M. A. Reed, C. Zhou, C. J. Muller, T. P. Burgin, and J. M. Tour, Science 278, 252 (1997).

${ }^{3}$ K. Stokbro, J. Taylor, M. Brandbyge, J. L. Mozos, and P. Ordejon, Comput. Mater. Sci. 27, 151 (2003).

${ }^{4}$ B. Xu and N. J. Tao, Science 301, 1221 (2003).

${ }^{5}$ R. Stadler, K. S. Thygesen, and K. W. Jacobsen, Phys. Rev. B 72, 241401(R) (2005).

${ }^{6}$ L. Venkataraman, J. E. Klare, C. Nuckolls, M. S. Hybertsen, and M. L. Steigerwald, Nature (London) 442, 904 (2006).

${ }^{7}$ F. Chen, X. Li, J. Hihath, Z. Huang, and N. Tao, J. Am. Chem. Soc. 128, 15874 (2006).

${ }^{8}$ J. Reichert, R. Ochs, D. Beckmann, H. B. Weber, M. Mayor, and H. v. Löhneysen, Phys. Rev. Lett. 88, 176804 (2002).

${ }^{9}$ J. Clayden, N. Greeves, S. Warren, and P. Wothers, Organic Chemistry (Oxford University Press, Oxford, 2001).

${ }^{10}$ L. Venkataraman, Y. S. Park, A. C. Whalley, C. Nuckolls, M. S. Hybertsen, and M. L. Steigerwald, Nano Lett. 7, 502 (2007).

${ }^{11}$ X. Xiao, B. Xu, and N. J. Tao, Nano Lett. 4, 267 (2004).

${ }^{12}$ J. M. Soler, E. Artacho, J. D. Gale, A. Garcia, J. Junquera, P. Ordejón, and D. Sánchez-Portal, J. Phys.: Condens. Matter 14, 2745 (2002).

${ }^{13}$ J. P. Perdew, K. Burke, and M. Ernzerhof, Phys. Rev. Lett. 77, 3865 (1996).

${ }^{14}$ F. Chen, J. Hihath, Z. Huang, X. Li, and N. J. Tao, Annu. Rev. Phys. Chem. 58, 535 (2007).

${ }^{15}$ S. Y. Quek, L. Venkataraman, H. J. Choi, S. G. Louie, M. S. Hybertsen, and J. B. Neaton, Nano Lett. 7, 3477 (2007).

${ }^{16}$ A. M. Bratkovsky and P. E. Komilovitch, Phys. Rev. B 67, 115307 (2003).

${ }^{17}$ K. S. Thygesen and K. W. Jacobsen, Chem. Phys. 319, 111 (2005). 
${ }^{18}$ M. Strange, I. S. Kristensen, K. S. Thygesen, and K. W. Jacobsen, J. Chem. Phys. (to be published), http://arxiv.org/abs/0709.4162.

${ }^{19}$ Z.-L. Cai, K. Sendt, and J. R. Reimers, J. Chem. Phys. 117, 5543 (2002).

${ }^{20}$ A. Filippetti and N. A. Spaldin, Phys. Rev. B 67, 125109 (2003).

${ }^{21}$ C. D. Pemmaraju, T. Archer, D. Sánchez-Portal, and S. Sanvito, Phys. Rev. B 75, 045101 (2007).

${ }^{22}$ J. B. Neaton, M. S. Hybertsen, and S. G. Louie, Phys. Rev. Lett. 97, 216405 (2006).
${ }^{23}$ Note that the SO shifts all occupied/unoccupied levels down/up in energy by the same amount, $\left|\Sigma_{0}\right|$, as discussed in Ref. 15. More details are given in the supplementary material of Ref. 24.

${ }^{24}$ See EPAPS Document No. E-JCPSA6-128-803811 for further details concerning the "scissors operator" and the calculation of the image potential. This document can be reached through a direct link in the online article's HTML reference section or via the EPAPS homepage (http:// www.aip.org/pubservs/epaps.html). 